C-A/AP/\#223

November 2005

\title{
Plasma Lens for Super Neutrino Beam at BNL and Other Applications
}

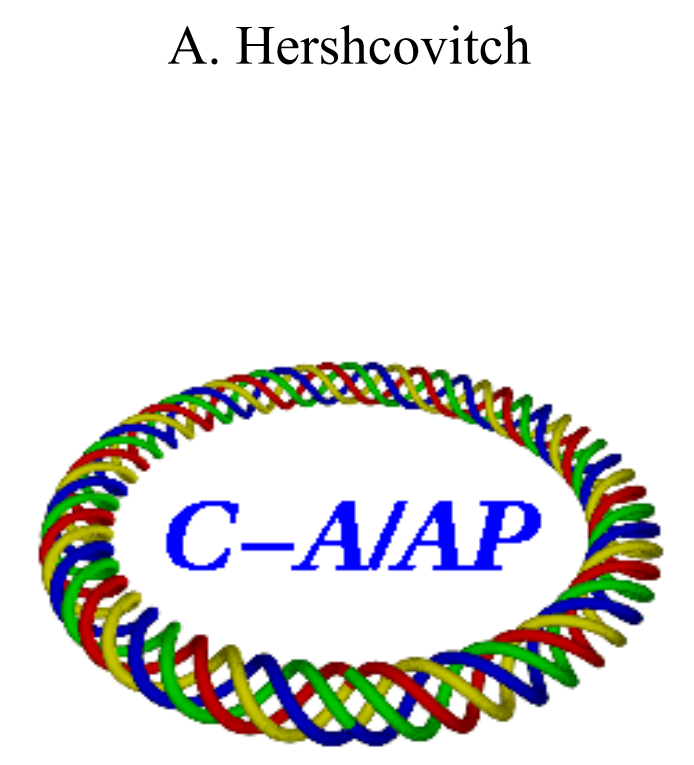

Collider-Accelerator Department Brookhaven National Laboratory Upton, NY 11973 


\title{
Plasma Lens for Super Neutrino Beam at BNL and Other Applications
}

\begin{abstract}
Preliminary ideas for novel pion capturing and focusing schemes are presented in this note. Although extensive simulations are needed before any conclusions can be drawn, a basic argument (plasma lenses can support larger currents; can shape discharge profile) is presented that suggests that $Z$ pinches and spark channels deserve a further, more serious consideration for possible use in the proposed BNL super neutrino facility. A Z pinch or a Z channel combined with an intense relativistic electron beam, or the use of inverse pinch configurations to capture and focus both positive and negative pions into separate regions in space is an intriguing possibility that may benefit the muon collider.
\end{abstract}

\section{INTRODUCTION}

In many areas of research involving charged particle beams, various methods of magnetic focusing have been employed to enhance the flux of charged particles from a divergent source such as a production target,[1] or to confine ions emerging from the cross-over region of an ion diode to betatron oscillation for propagation to a small target a few meters away.[2] The method of choice for focusing of high energy charged particles, produced in nanosecond to microsecond bursts, that need to be transported for a distance of a meter or more has been the use of azimuthal magnetic fields that pull the particles radially inward as a consequence of the Lorentz force. Large currents that are oriented along the desired flight path of the charged projectiles usually generate strong azimuthal magnetic fields. Therefore, devices with large axial current can be utilized as lenses.

Lithium lenses[3,4] and focusing horns[1,5] have been used in high energy physics research, while various spark and $Z$ channels were developed for fusion experiments.[2,6,7,8] Spark and $\mathrm{Z}$ channels shall be referred to as plasma lenses in this note, even though in high energy physics research this term was used in reference to lithium lenses and similar lenses in which the lithium was replaced by high pressure gases. In all applications, the purpose of these lenses is to focus and to facilitate propagation of charged particle beams with a minimum of beam absorption and scattering.

Although some features vary from experiment to experiment, there are a number of common requirements for all these lenses including the BNL Super Neutrino Facility:

1) Very large axial electrical currents (Mega-amps) must be generated and sustained.

2) The magnetic fields generated by these currents should capture the largest number of parent pions.

3) The lens medium should have lowest density possible to minimize pion absorption and scattering.

4) The lens must endure high mechanical and thermal stresses caused by high currents and EM fields.

5) The lens must survive prolonged exposure to radiation. 
6) The lens should minimize neutrino background during anti-neutrino beam runs (signal purity);

7) A cost-effective, power-efficient lens is desirable.

For generating large neutrino beams, high-energy pions must be captured and maintained as a beam until they decay. Description and comparison of the various lenses, as well as integrated plasma lens/target are presented in this note.

\section{DEVICES GENERATING LARGE AXIAL CURRENTS}

Interest in this type of charged particle focusing is varied and many of the broad number of applications require customized lens configuration. Lens choice in this note, however, is done based on applicability to neutrino generation.

A. A horn system is a hollow coaxial structure of conductors through which large currents (100s of kA) flow to generate the focusing magnetic fields.[1,5] These conductors must be shaped such that the magnetic field integral increases with radius, thus providing focusing for particles located at radii larger than that of the inner horn inside radius. Requirements on the inner horns are extremely demanding: they have to withstand very large thermal and mechanical stresses; yet, they must be fabricated from light elements to minimize particle losses.

B. A lithium lens consists of a cylindrical conductor (made of lithium) through which a large axial current is induced to generate an azimuthal magnetic field.[3,4] Unsuccessful attempts were made to replace lithium with compressed gases or aluminum. Even though lithium has very low mechanical strength, and it is very reactive chemically, it remains the conductor of choice since it is light (to minimize loss of particles), and conducts electricity well. It must, however, be kept under high pressure in a strong container made of a chemically compatible material like titanium or stainless steel.

C. Spark (or $\mathbf{Z}$ ) channels are plasma transport channels, characterized by large currents (100s of kA), which have been developed to transport (and focus) intense beams of light ions (with currents of 100s of $\mathrm{kA}$, and energies of a few $\mathrm{MeV}$ ) over distances of up to 5 meters.[8]

These channels consists of two annular plates (or rings) placed in a vacuum chamber. These plates are biased to serve as anode and cathode of the discharge, their spacing determines the channel length. The vacuum chamber is usually filled to a pressure of as low as a few Torr to as high as 40 Torr with either a light or a heavy gas. After an appropriate bias (10s of kV) is applied to the plates and an appropriate gas fill of the vacuum chamber, a discharge can be initiated with either an exploding wire or a laser pulse. This initial discharge preionizes and heats the gas. After the heated gas expands and rarefies on axis, a four-fold reduction in gas density on axis occurs with a corresponding ten-fold reduction in the breakdown voltage. Once breakdown occurs, the hot expanding channel acts as a piston compressing the gas outside the channel. Choice of an appropriate gas fill determines the channel expansion rate that must accommodate the current rise time (which is in turn determined to a large extent by the circuitry).

A large variety of these channels have been made, and an even larger variety is possible.[9] Pulse lengths of $10 \mathrm{~s}$ of nsec at a repetition rate of $500 \mathrm{~Hz}-1 \mathrm{kHz}$ have been 
generated, as well as three microsecond long pulses at lower repetition rates. Hundreds of kA of discharge currents have been attained. Channel radii from $1 \mathrm{~cm}$ to over $10 \mathrm{~cm}$ were reported (larger radii are easy to generate, it is next to impossible to generate channels with radii that are below $1 \mathrm{~cm}$, which is desired for inertial confinement fusion). Another feature of these channels, which adds to their versatility, is the ease with which the direction of the discharge current, and hence the focusing magnetic field can be changed (by externally changing the applied bias on the plates). Thus, a single channel can alternately be used to focus positive and negative particles on a shot-to-shot basis.

D. Mega-Ampere electron beams. Any type of axial current generates azimuthal magnetic fields. Electron beam currents that are in the mega-Ampere range have been generated by diodes. Although most of these diodes operate with pulses that are in the nsec range, some diodes have operated with pulse lengths of up to 2 microseconds. More conventional electron guns (some with plasma cathodes) can also be stacked up (or even scaled up) to yield 100s of kA to $1 \mathrm{MA}$ of current. Although in most practical applications (pulse lengths of 100 microseconds or longer), current densities in beam forming gun structures are limited to $100 \mathrm{~A} / \mathrm{sq} . \mathrm{cm}$ due to voltage breakdown effects. Much larger current densities are possible in devices operating with pulse lengths that are sufficiently short (no more than a few microseconds, i.e., shorter than an arc propagation time).

A hybrid system in which an electron beam is launched and is propagated through a plasma channel can be a very attractive option, since it is possible that neither technique may need to be "pushed" to its technological limit to reach resultant axial currents exceeding 1 MA that are 1 meter long. Hollow-beam electron guns may be particularly suitable for such an application due to their larger perveance and enhanced stability in addition to the obvious advantage of their hollow structure.

E. $\quad \underline{Z}$ pinches. A $Z$ pinch involves a sudden compression of low-density plasma by means of a large discharge current that lasts for a few microseconds. It bears some superficial similarity to a spark channel in that a discharge is formed between two end plates, but their plasma properties are very different. Its fill pressure is below a milli-Torr. First, a low-density, low-temperature plasma is created by rf or exploding wires. Second, a large voltage is applied to the end plates that drives a very large axial current that compresses the plasma due to an inward acceleration of a surface current shell (just opposite to what occurs in spark channels). In a series of experiments with magnetized $Z$ pinches, $2 \mathrm{MA}$ were reached for a length of 0.8 meters with an axial magnetic field of 1.5 Tesla.[10] Present day Z-pinch research involves discharge currents of $10 \mathrm{MA}$ over a few centimeters have been reached in a rather expensive system.[11]

\section{LENS OPTIONS}

Presently, a horn system is being considered for pion capturing. The first focusing lens for the Super Neutrino beam is a $250 \mathrm{kA}$ horn surrounding the $6-\mathrm{mm}$ radius, $80 \mathrm{~cm}$ long carbon target.[12] State-of-the-art performance for a horn is $300 \mathrm{kA}$. It can be made with a large enough diameter, which makes it well suited for these experiments. But, it has technical difficulties and increase in current leads to further technical difficulties of an engineering task that is already very demanding. Nevertheless, the major disadvantage is that horns do not capture pions with velocities that are at very small angles to target axis. 
Peak performance of a lithium lens was $750 \mathrm{kA}$,[13] but typical top performance is at the $500 \mathrm{kA}$ level. The radius of a lithium lens is $1 \mathrm{~cm}$ or smaller. Therefore, the magnetic field at a distance of about $10 \mathrm{~cm}$ from the lens axis (where it is most important for focusing) is an order of magnitude lower than at the lens radius. Thus, for this particular application, a lithium lens is not very attractive.

Currents of 10 MA have been generated in $\mathrm{Z}$ pinches for lengths of a few centimeters.[11] But, the discharge collapses to a very small diameter. It is possible to prevent the pinching effect and shape the plasma and current profile with a large axial magnetic field, which together with a stabilizing wall also allow for longer discharges with even larger current, in addition to the larger radii. However, no such research was done for 50 years, since the main objective of a pinch is to heat and compress plasma for the purpose of attaining thermonuclear fusion. The last large-scale attempt at magnetized Z-pinch fusion resulted in a 0.8-meter long 6$\mathrm{cm}$ radius discharge with a current of $2 \mathrm{MA}$ for $250 \mu \mathrm{sec}$ duration (1.5 Tesla axial magnetic field). But, the ion temperature was only $50 \mathrm{eV}[10]$ (disastrous for fusion). Nevertheless, forty years ago a 1.5-meter long, 40-cm diameter Z-"pinch" lens, with a current of $500 \mathrm{kA}$ for $15 \mu \mathrm{sec}$ duration was successfully used in an AGS experiment.[14] This lens performed very well until its ceramic liner broke and was not replaced since the experiment was close to its conclusion.[15] Since then various special kevlar, fiberglass, and carbon epoxy liners and insulators were developed for radiation generating machines (used in weapon research) that are durable under extremely intense radiation.

Spark (or Z) channels were developed to transport and focus light ion beams into small DT pellets and drive fusion microexplosions. Hence, these channels were optimized for light ion inertial confinement fusion. Currents of up to $200 \mathrm{kA}$ were achieved (sufficient for focusing 1 $\mathrm{MeV}$ carbon ions in less than 0.4 meter). The longest channel generated was 5 meters long.[8] This channel was 10 centimeters in radius; it had a current of $50 \mathrm{kA}$ (more than what is needed to focus any conceivable light ion beam for fusion).

The latter two, along with electron beams, possess all the features listed in the introduction, which are required for a BNL Super Neutrino Facility capturing lens. Additionally, they have the advantage of higher currents, whose profiles can be shaped.

\section{NOVEL LENS/TARGET FOR PION CAPTURE}

Plasma lenses offer many advantages over other focusing methods. Current levels of tens of mega-amps are carried routinely in some plasma devices, while current levels in conventional horns and lithium lenses cannot exceed a few hundred kilo-amps. In addition to be able to support larger currents than other lenses, plasma lenses can have their discharge profiles shaped for optimized pion capture. Unlike the presently considered horns, plasma lenses can capture and deflect pions with velocities that are at very small angles to target axis. In plasma lenses, current is carried in low-density ionized gases, while in other lenses the current is carried in solid matter or in liquids. Except for a couple of small electrodes, the bulk of a plasma lens is essentially empty space. Consequently, attenuation of generated particles in plasma lenses is minimal. Since the major component of a plasma lens is ionized gas, the bulk of the lens is impervious to radiation and thermal damage. As mentioned in the previous paragraph, forty years ago, a 1.5meter long, 40-cm diameter "Z-pinch" lens, with a current of $500 \mathrm{kA}$ for $15 \mu \mathrm{sec}$ duration was successfully used in an AGS experiment. This lens performed very well until its ceramic liner broke and was not replaced since the experiment was close to its conclusion. Since then, various 
special kevlar, fiberglass, and carbon epoxy liners and insulators have been developed for radiation generating machines (used in weapons research) that are durable under extremely intense radiation. Also, dramatic improvements in capacitors have occurred during the past twenty five years that resulted in a quantum jump in this important technology.

Although horn technology is mature and horns are reasonably suited to neutrino beam generation, plasma lenses have many advantages over horns that cover all seven points of an "ideal lens." As noted in the previous paragraph, plasma lenses are better than horns for each of the first five points. Since plasma lenses can capture negatively charged pions while deflecting positively charged pions with trajectories that are at very small angles to the target axis (unlike present horns), the neutrino signal can be minimized during anti-neutrino running (point 6). Presently (based on the use of horns), $15 \%$ of the beam signal is neutrinos during anti-neutrino runs (while; in case of neutrino run, anti-neutrinos constitute $4 \%$ of the signal). Finally on point 7, horns use more power and contain more material (cost more). As horns heat-up, their resistance (and power consumption) increases while the plasma resistance drops during the pulse (after 1 nsec Voltage drops from about $20 \mathrm{KV}$ to a few Volts).

\section{A. The Plasma Lens as a Neutrino Beam Focusing System}

A key requirement for a successful future neutrino program will be a very high-power (Megawatt-class) source of high-energy protons, together with a very efficient pion-focusing system downstream of the primary beam production target. To maximize the flux of neutrinos into an experimental neutrino detector located far from the beam source, as required by a Very Long Baseline Neutrino Oscillation (VLBNO) experiment, the maximum number of high-energy pions from a primary proton target must be captured and magnetically focused into a directed beam before they decay into muons and neutrinos in the decay region of the source. Since the plasma lens possesses more of the ideal pion focusing and capturing characteristics for maximizing neutrino beam signal at the detector than other lenses, two options are considered and shown in figures 1 and 2. In the first, some or most of the current is pulsed through the target, while in the second option the current channel straddles and runs outside the target.

To pulse large currents through the outer part of the $6-\mathrm{mm}$ radius, $80 \mathrm{~cm}$ long carbon target, high conductivity graphite like quasimonocrystal graphite, which has resistivity that is 34 times larger than copper (most graphites are 1000 times more resistive than copper), should be utilized as target material. Part of the current can be carried by plasma outside the target; this way target heating by current is minimized. The current continues in a Z-pinch downstream following the target to capture pions that have trajectories that are at small angles to the target axis, and would not be captured by the currently pursued horns.

Figure 1 is a schematic of a combined lens/target configuration. Current can be fed at an electrode located some point downstream from the beginning of the target where pion capturing is needed. Feed point can be, if necessary, varied in situ to optimize neutrino flux. The target is followed up by a plasma lens, which has an additional current feed to facilitate higher (or different) current in the plasma lens. It is controlled by the second electrode. This section of the plasma lens is immersed in a solenoidal magnetic field to facilitate its current profile shaping. Current profile can be shaped to optimize pion capture.

Driving current through the production target increases its heat load, which is already a

serious issue. Room temperature resistance of a carbon target made of high conductivity graphite 
like quasimonocrystal graphite is about $6 \times 10^{-3} \mathrm{Ohm}$ at room temperature. Considering worst case scenario of $300 \mathrm{kA}$ through the target (horn's current is only $250 \mathrm{kA}$ ) for $3 \mu \mathrm{sec}$, energy deposited in the target is about $1.7 \mathrm{~kJ}$. By comparison, proton energy deposited per pulse in the target is $7.3 \mathrm{~kJ}$, i.e., deposited electrical energy increases target heat load by $23 \%$. Reducing the current to $250 \mathrm{kA}$ results in $1.18 \mathrm{~kJ}$ electrical deposited (16\% of beam energy deposition), and as the target heats up, its resistivity decreases. Depending of simulation results, chances are that per pulse electrical energy deposited in the target will be only a small fraction of the $1.7 \mathrm{~kJ}$ worstcase scenario, since much of the current will flow in the plasma outside the target.

A better approach may be to drive the current exclusively outside the target, as shown in figure 2. The plasma straddles the target, which provides the necessary stability against kink and sausage instabilities that tend to plague unstabilized Z-pinches. At current levels of $300 \mathrm{kA}$ or less, the carbon target should not be affected by the Z-pinch compression. Following the target, the rest of plasma lens consists of a magnetized Z-pinch that is identical to the figure 1 configuration. Plasma size and shape, as well as the wheel like electrode design can be chosen to maximize neutrino flux.

In both of the above cases, the effect of the magnetic fields generated by current flowing through spokes of the wheel shaped electrodes should be minimal, since the magnetic field is symmetric in magnitude but opposite in direction about each spoke. Hence charged particles approaching a spoke are deflected one way, while being subjected to opposite deflection as they move away from the spoke.

\section{B. $\quad$ Capturing Positive and Negative Pions with Inverse Pinch Configurations}

A configuration that has the potential of capturing both positively charged and negatively charged pions is shown in figure 3 . The configuration is characterized by a center conductor (or a discharge) carrying current in one direction, and cylindrical plasma sheet carrying a current in the opposite direction. Azimuthal magnetic field generated by the inner current prevents collapse, due to pinching of the outer current shell. In fusion devices the gap between the two currents is of the order of 10's of cm, but there is much flexibility. Current levels of MA's have been generated in similar fusion devices known as RFP's (reversed field pinches). A second lens with this configuration can be added down stream and additional current can be driven in either direction. This section can be immersed in a solenoidal magnetic field to enhance stability and to facilitate current profile shaping.

Positively charged pions can be captured by inner current generated magnetic field, which deflects negatively charged particles outward. But, due to the forward momentum of the parent protons, the negatively charged pions retain forward motion. Once reaching the outer current, these negatively charged pions are subjected to a magnetic field that exerts an inward Lorentz force and captures negatively charged pions. Simulations of pion trajectories are needed to design optimum configurations.

RFP's can be used to capture and focus pions and muons in the proposed muon collider. Depending on what the pion generating target is, current can be driven through the target, with the return current cylinder straddling the target. A second lens with this configuration can be added down stream and additional current can be driven in either direction. This section can be immersed in a solenoidal magnetic field to enhance stability and to facilitate current profile shaping. The return current cylinder can be easily shaped into a cone-like configuration if needed (like the horns). 
The advantages of this pion capturing approach are:

1. Much larger quantity of pions can be, in principle, captured.

2. Captured positive and negative pions (as well as muons) are guided into different spatial regions. For example, positive pions and muons trajectories form a central channel, while the negative particles have trajectories in the shape of a hollow cylinder (like a halo). It's unlike the presently considered solenoid, which focuses both species into the same spatial region.

3. Ionization cooling can easily follow capture; negative pions can be focused into a channel following ionization cooling.

Neutrino flux simulations are needed to show the viability of any scheme. And many other experiments that require focusing and capturing of charged particles, like g-2, may benefit from a plasma lens.

\section{ADDITIONAL POSSIBILITIES}

Concepts discussed up to this point are based on either experimentally proven devices or on small variations of these devices, i.e., devices requiring some modifications. More speculative ideas are mentioned in this short section.

Previously discussed current carrying configurations are based on discharges in basically vacuum devices, or at least start with below atmospheric pressure. But present BNL Super Neutrino Facility design is based on cooling the target with helium gas at a pressure of 5 atmospheres. High pressure vortex stabilized current channels can be utilized instead of low pressure electrical discharges like Z-pinches. Figure 4 is a photo of a vortex-stabilized discharge that is extended from a plasma window. Vortex driven by above atmospheric pressure stabilizes the figure 4 helium discharge, which is steady state. Applying the helium cooling as a vortex can provide both target cooling and focus current discharge stability.

Similarly spark channels can be, in principle, generated in high pressure like lightening. But, unlike lightening, which is unstable walled boundary like a tunnel (or a large pipe) can stabilize the discharge. The price for having high pressure discharges is the large voltages are needed to sustain the arcs.

Hollow spark channels can also be made in principle in both low and high pressures. These channels can be used to drive the inverse currents for capturing pions with charge opposite to those captured by central currents.

\section{CONCLUSIONS}

Discharges described in this note like Z-pinches or spark channels can, in principle, provide for improved pion capture and focusing, since larger currents with current profiles optimized for pion capture can be generated. An unlike horns, large currents can be sustained along the target axis to capture pions that have velocities with very small angles to target axis. If necessary desired currents can be generated or augmented with electron beams (including hollow electron beams). Hybrid systems of $\mathrm{Z}$ pinches, electron beams, and/or spark channels, are interesting ideas to pursue.

Configurations that can in principle capture and focus both positive and negative pions have clear advantages. Inverse pinches are obviously suited as possible lens. But, hybrid systems 
involving relativistic electron beams may prove to have other advantages (electrons scatter less and cause less erosion).

Further evaluation requires an iteration process of simulations and of lens/target design to further explore concepts presented in this note.

Notice: This manuscript has been authored by Brookhaven Science Associates, LLC under Contract No. DE-AC02-98CH1-886 with the US Department of Energy. The Untied States Government retains, and the publisher, by accepting the article for publication, acknowledges, a world-wide license to publish or reproduce the published form of this manuscript, or others to do so, for the United States Government purposes.

\section{REFERENCES}

1. M. Giesch, et al., NIM $\underline{20}, 58$ (1963).

2. C.L. Olson, J. Fusion Energy 1, 309 (1982).

3. G. Dugan, et al., IEEE Trans. Nucl. Sci. NS-30, 4, 3660 (1983).

4. A. Ijspeert and P. Sievers, CERN SPS/86-18 (ABT).

5. A. Carroll, et al., Proceedings of IEEE Particle Accelerator Conf. 1987, p. 1731.

6. J. Olsen, D. Johnson, and R. Leeper, Appl. Phys. Lett. 36, 808 (1980).

7. J. Olsen and R. Leeper, J. Appl. Phys. 53, 3397 (1982).

8. P. Ottinger, et al., Proceedings of IEEE, $\underline{80}, 1010$ (1992).

9. C.L. Olson, private communication, 1994.

10. A series of papers (by Golovin, et al., Komelkov, et al., and Andrianov, et al.), presented at the second U.N. Conf. on Peaceful Uses of Atomic Energy, Vol. 31 and 32, Geneva, 1958.

11. Work by McDaniel, et al., D.L. Cook, private communication, 1994; Rahman, Wessel, and Rostoker, reported current levels of 5 MA in PRL 74, 714 (1995).

12. AGS Upgrade \& Super Neutrino Beam Facility, BNL Review June 10-11, 2004 (unpublished).

13. C. Hojvat, private communication, 1994.

14. E.B. Forsyth, L. Lederman, and J. Sunderland, IEEE Trans. Nucl. Sci., p. 872 (June, 1965).

15. E.B. Forsyth, private communication, 1995

\section{$\underline{\text { Figure Captions }}$}

1. Schematic of a lens based on current driven through the target followed by a magnetized Z-pinch (to also capture pions with velocities that are predominantly axial).

2. Schematic of a lens, in which a $Z$ pinch straddles the target followed by a magnetized Zpinch. Also well suited to capture pions with predominantly axial velocities.

3. Diagram of lens based on inverse pinch configuration with central current and cylindrical sheet current flowing in opposite directions. Current direction in the center is designed to capture positive pions, while the magnetic field generated by the hollow sheet current captures negative pions.

4. Photo of vortex stabilized plasma extended about one inch from a plasma window with vortex generated in the plasma window. 


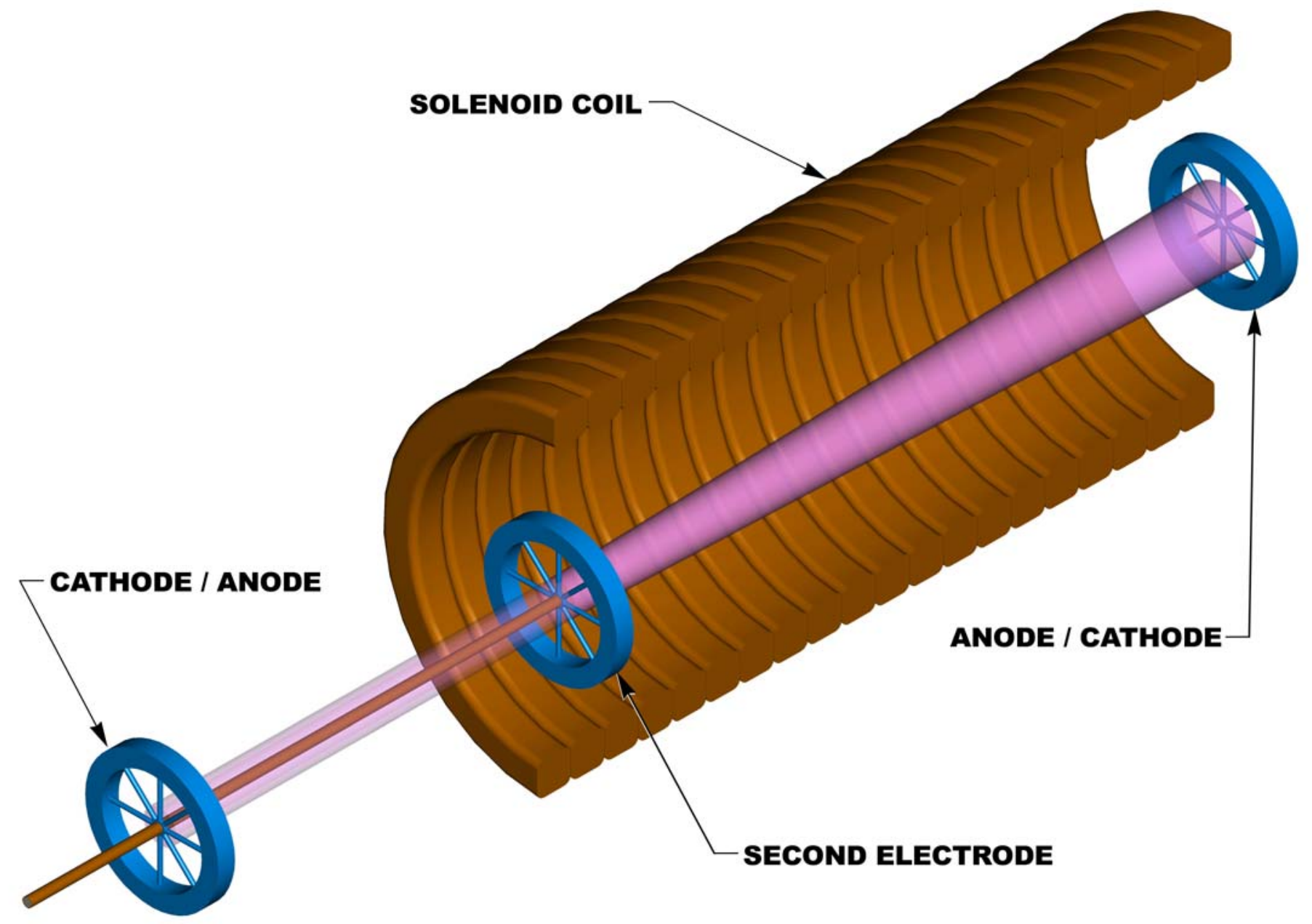

Figure 1 


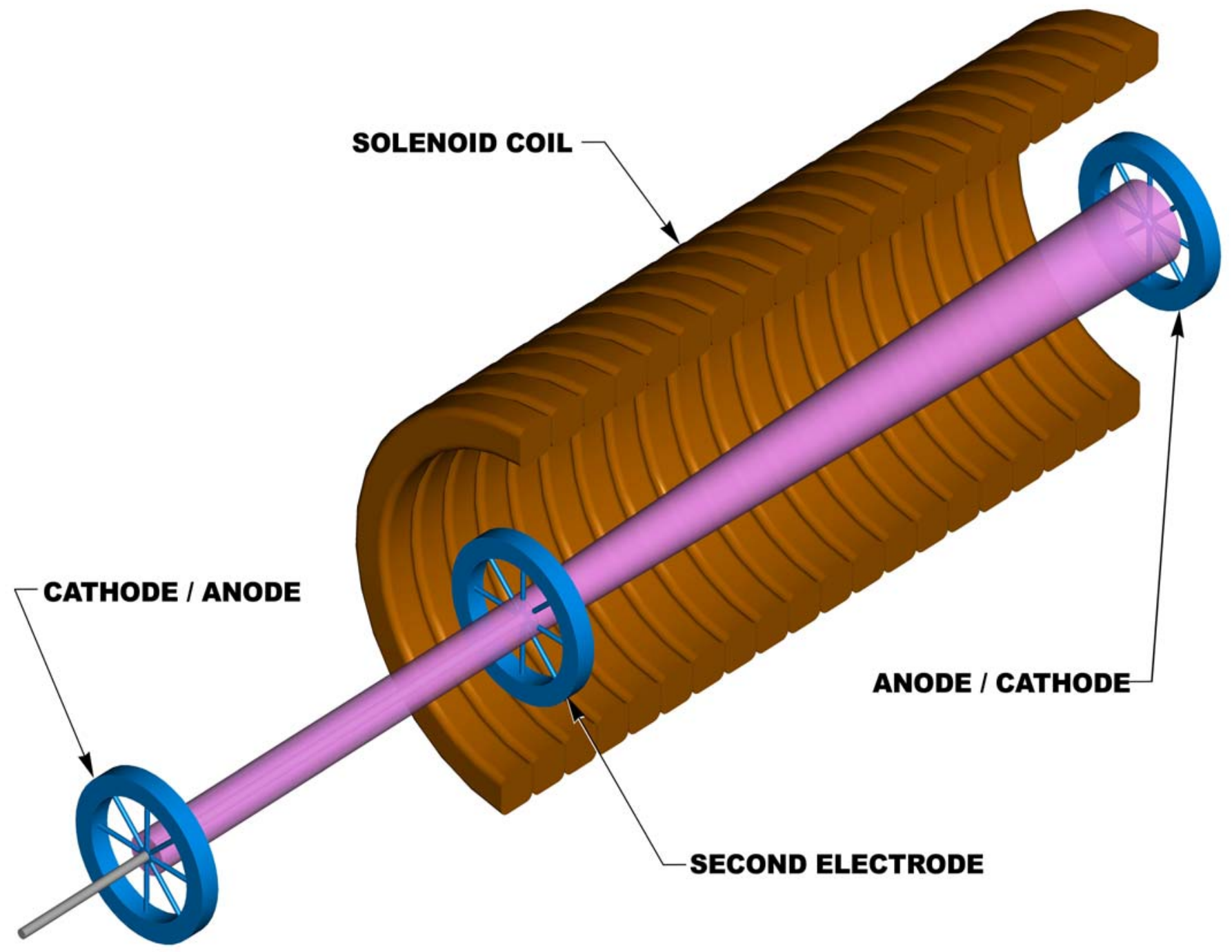

Figure 2 


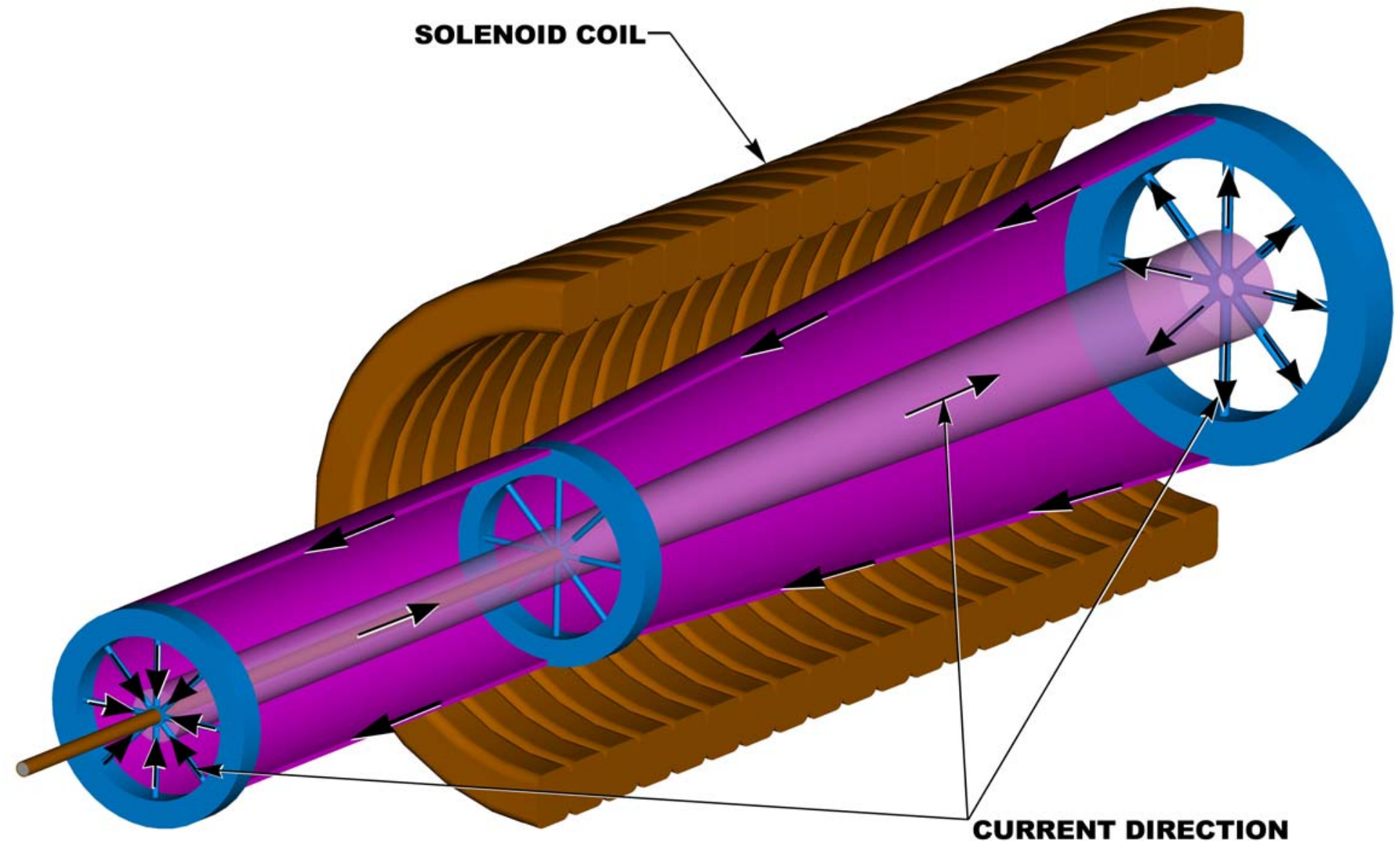

Figure 3 


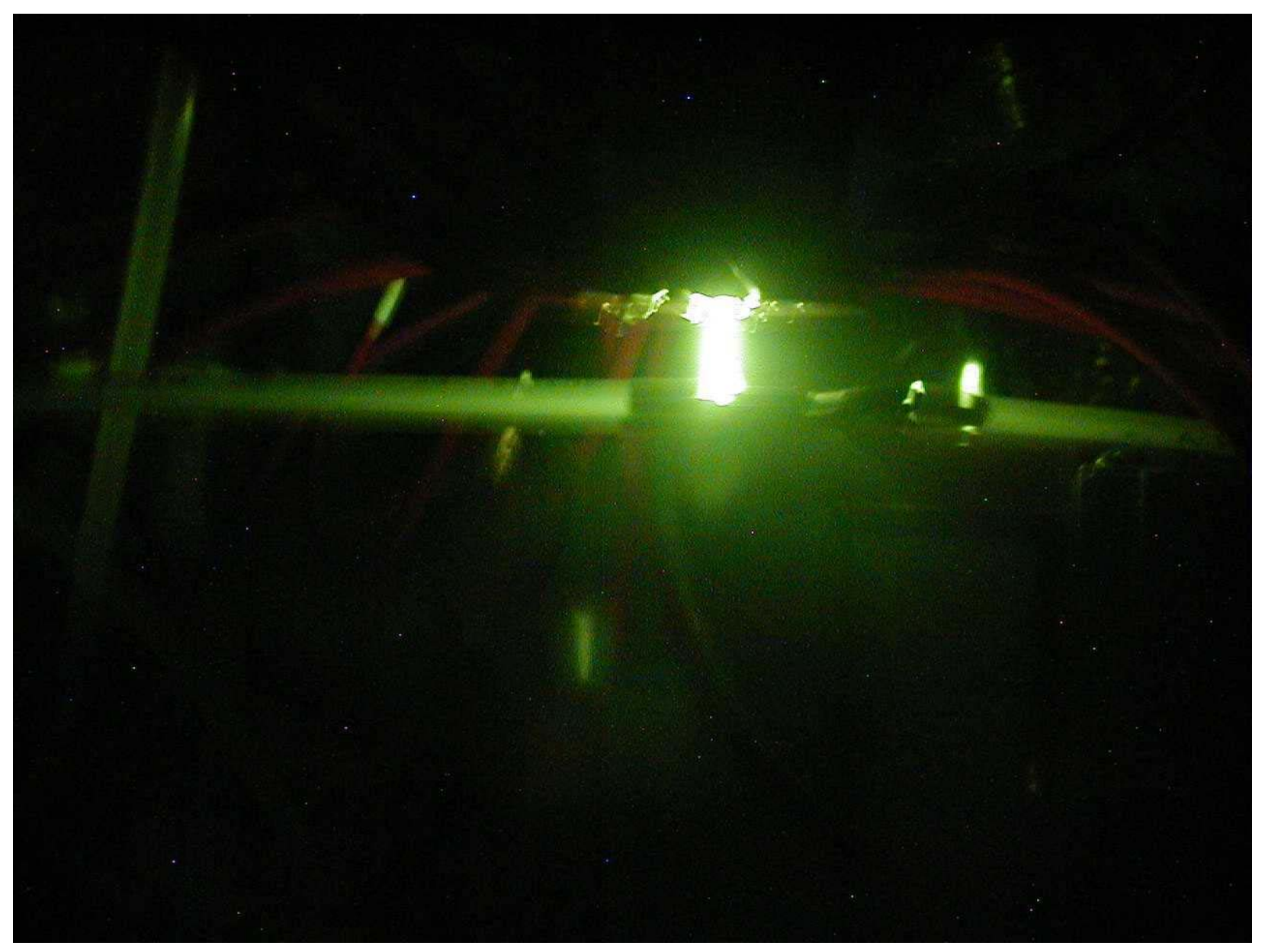

Figure 4 